Of the 217 assessments 109 were in SCCAI defined remission, 95 were in $\mathrm{PRO} 2$ defined remission and the two were highly contingent $(\mathrm{p}<0.0001)$. The sensitivity and specificity of disease activity assessments made with PRO2 vs SCCAI were 0.85 and 0.98 , respectively. The positive and negative predictive values were 0.98 and 0.87 , respectively.

Conclusions The PRO2 performs well when validated against an established clinical disease activity index (SCCAI), quality of life assessments and biochemical markers of disease activity. PRO2 assessments have the benefit of being more rapid to administer (comprising of only 2 items) than SCCAI (6 items), whilst providing similar and accurate evaluations of remission status.

\section{P144 THERAPEUTIC THRESHOLDS FOR GOLIMUMAB SERUM CONCENTRATIONS DURING INDUCTION AND MAINTENANCE: RESULTS FROM THE GO-LEVEL STUDY}

Mark Samaan*, Georgina Cunningham, Gokul Tamilarasan, Krystal Rawstron, Karim Hawash, Luisa Beltran, Shuvra Ray, Joel Mawdsley, Simon Anderson, Jeremy Sanderson, Zehra Arkir, Peter Irving. Guy's and St Thomas' Hospital, London, UK

\subsection{6/gutjnl-2020-bsgcampus.219}

Introduction The exposure-response relationship associated with the use of golimumab for UC was demonstrated in the PURSUIT trial. Significant associations between serum golimumab concentrations (SGC) and favourable outcomes were observed during both induction and maintenance therapy. However, data regarding the optimal therapeutic SGC threshold is limited and therefore, recent AGA guidance made no recommendation in this regard.

Methods GO-LEVEL was an open label, phase IV, investigatorinitiated study (NCT03124121) which included a prospective cohort of UC patients commencing golimumab induction therapy, as well as a cross-sectional cohort receiving maintenance treatment (>18 weeks from initiation).

Patients commencing induction all had disease activity objectively confirmed and were evaluated at weeks 6, 10 and 14. Patients receiving maintenance therapy were recruited either at the point of flare, or during stable remission. Clinical disease activity was evaluated using SCCAI and PRO2, and biochemical activity using FC and CRP. Combined clinical-biochemical remission was defined as SCCAI less than 3 and FC less than $250 \mathrm{ug} / \mathrm{g}$.

SGC and anti-golimumab antibodies (ADA) were measured using a drug sensitive ELISA (LISATRACKER, Theradiag).

Mann-Whitney U was used to compare groups, ROC curve analysis to identify therapeutic thresholds, Spearman's rank coefficient $\left(r_{s}\right)$ for correlations and Cochrane-Armitage test for quartile data.

Results Thirty-nine patients were included in the induction cohort, of whom 15 (38\%) achieved combined remission at week 6. The median SGC of those in combined remission was significantly higher than those who were not (5.0 vs 3.1 $\mathrm{ug} / \mathrm{ml}$, respectively, $\mathrm{p}=0.03$ ). ROC curve analysis demonstrates $3.8 \mathrm{ug} / \mathrm{ml}$ as the optimal therapeutic threshold to achieve combined remission at week 6 (sensitivity 0.71, specificity 0.65, AUC 0.72). Significant, inverse correlations were observed between week $6 \mathrm{SGC}$ and PRO2, CRP and FC $\left(\mathrm{r}_{\mathrm{s}}-\right.$ $0.41 \quad(p=0.01), \quad-0.47 \quad(p=0.004)$ and $-0.40 \quad(p=0.02)$, respectively).

Sixty-four patients were included in the maintenance cohort; $32(50 \%)$ were in combined remission, 32 were not.

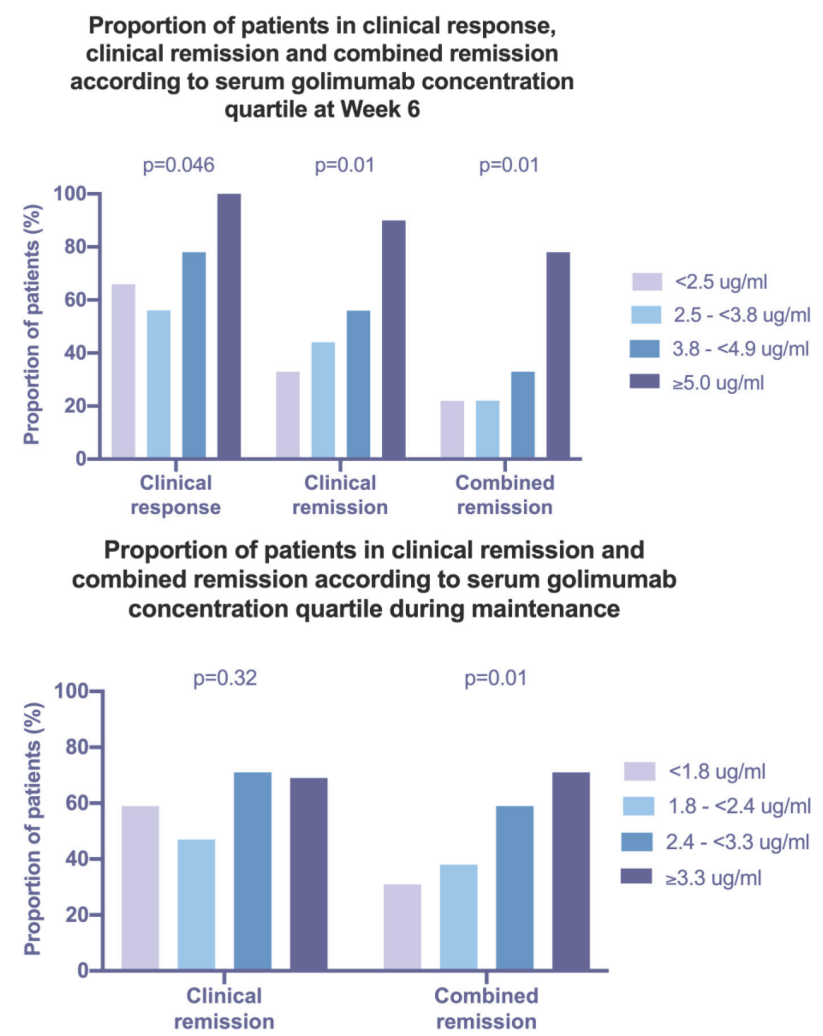

Abstract P144 Figure 1 SGC quartile analyses at week 6 during induction (left panel) and during maintenance therapy (right panel)

The median SGC of those in combined remission was significantly higher $(3.0$ vs $2.1 \mathrm{ug} / \mathrm{ml}$, respectively, $\mathrm{p}=0.003)$. ROC curve analysis demonstrates $2.4 \mathrm{ug} / \mathrm{ml}$ as the optimal therapeutic threshold to achieve combined remission (sensitivity 0.73 , specificity 0.66, AUC 0.71).

SGC quartile analyses (figure 1) demonstrated significant trends to higher rates of combined remission with greater exposure during both induction $(\mathrm{p}=0.01)$ and maintenance $(\mathrm{p}=0.01)$.

No AGA were detected in either cohort.

Conclusions GO-LEVEL offers further evidence of the exposure-response relationship with golimumab. Clinicians may consider using therapeutic drug monitoring to optimise golimumab dosing aiming to achieve our suggested SGC therapeutic thresholds of $3.8 \mathrm{ug} / \mathrm{ml}$ at week 6 and $2.4 \mathrm{ug} / \mathrm{ml}$ during maintenance.

\section{P145 BIOLOGICS ARE INFERIOR TO SURGERY IN ISOLATED INTERNAL PENETRATING CROHN'S DISEASE: A SINGLE CENTRE EXPERIENCE}

${ }^{1}$ Gregory Sebepos-Rogers*, ${ }^{2}$ Nader Al-Shakarchi, ${ }^{2}$ Tanvi Khetan, ${ }^{1}$ Sara McCartney, ${ }^{1}$ Stuart Bloom, ${ }^{1}$ Edward Seward, ${ }^{1}$ Roser Vega, ${ }^{1}$ Faroog Rahman, ${ }^{1}$ Shameer Mehta. ${ }^{1}$ University College Hospital, Department of Gastroenterology, London, UK; ${ }^{2}$ University College London, School of Medicine, London, UK

\subsection{6/gutjnl-2020-bsgcampus.220}

Introduction $50 \%$ of patents with Crohn's disease (CD) develop fistulae, resulting in significant morbidity. Isolated internal penetrating CD (IPCD), without enterocutaneous manifestations, is the second most prevalent fistulating phenotype after perianal $\mathrm{CD}(\mathrm{Schwartz}$ et al., 2019) yet the management 
of isolated IPCD remains poorly characterised in therapeutic trials. This study aims to assess outcomes of IPCD at a large, tertiary IBD centre.

Methods All adult patients with penetrating CD were screened from imaging reports and corroborated with radiology meeting, surgical and biologic databases between January 2016 and April 2019. Concurrent perianal or enterocutaneous fistulation was excluded from analysis. Complete, partial or no resolution of fistula was an assessed composite of imaging, symptoms and nutritional independence(Samimi et al., 2010). Statistics: categorical variables, Fisher's exact.

Results 29 patients had IPCD diagnosed between 2008 and 2018. 28\% had undergone prior gastrointestinal surgery. At fistula diagnosis, 41\% had prior biologic exposure including 6 on their third line biologic, and $66 \%$ on a previous or current immunomodulator. Only $4(14 \%)$ were on a current biologic.

IPCD was diagnosed at median 62 months [IQR 24-117] after IBD diagnosis with the majority enterocolonic (47\%) and enteroenteric (27\%) in configuration. 69\% had documented distal obstruction or stenosis, $7 \%$ had a confirmed abscess.

$52 \%, 24 \%$ and $24 \%$ underwent medical, surgical or no additional intervention, respectively, as primary management of their IPCD. Compared with no intervention, only the presence of symptoms was predictive of medical (14\% vs $93 \%$, $p=0.0006)$ or surgical $(14 \%$ vs $100 \%, p=0.0047)$ intervention, whereas prior surgery, current or prior biologic therapy, IPCD configuration were not. Of medically treated IPCD, $73 \%(11 / 15)$ had a biologic at median duration 29 months with Adalimumab 1st line in 64\% (7/11) and Ustekinumab 2nd line in $67 \%(4 / 6)$.

There was a trend to greater complete or partial fistula resolution by surgery versus medical management $(86 \%(6 / 7)$ vs $47 \%(7 / 15), p=0.1649)$ and surgery versus biologics (86\% (6/ $7)$ vs $64 \%(7 / 11), p=0.596)$ at reassessment interval (17 [1243] vs 8 [7-17] months, respectively) (figure 1). Of medical
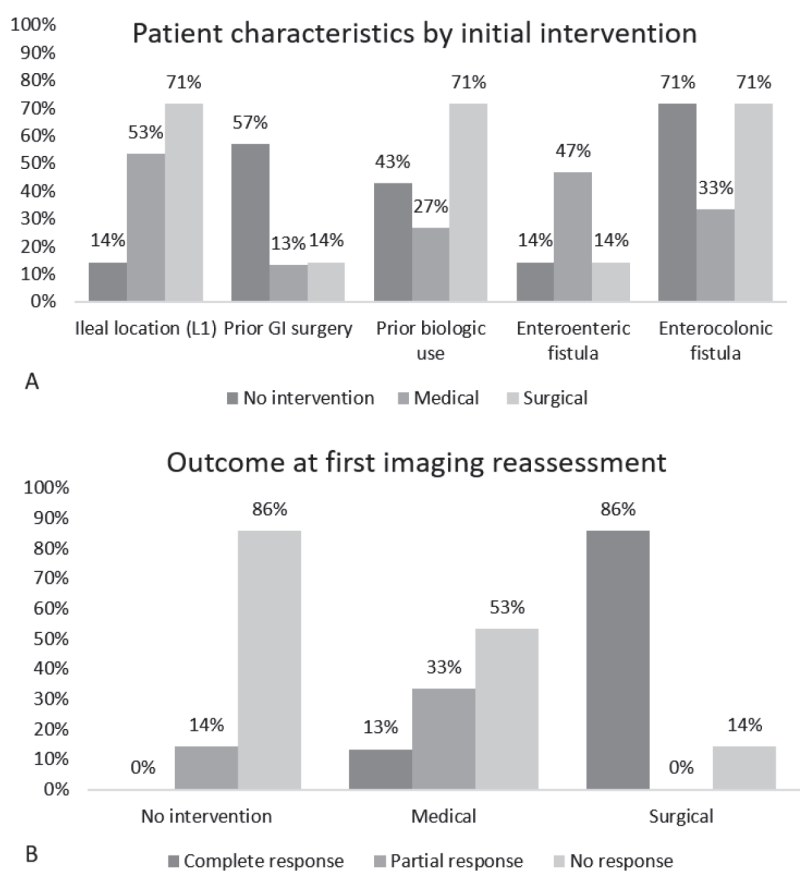

Abstract P145 Figure 1 Categorisation of intervention decisionmaking of IPCD by A. Key patient characteristics, B. Outcome at reassessment and no intervention cohorts, 53\% and 29\% eventually had surgery at median intervals 6 and 72 months.

Of the whole cohort, 59\% had surgery, in whom 35\% had a pre-operative biologic, $12 \%$ pre-operative parenteral nutrition (PN), 18\% post-operative PN (duration 14, 41, 8 months respectively), and $18 \%$ post-operative intra-abdominal septic complications within 30 days.

Conclusion Most patients in this cohort require surgery to achieve complete of resolution of IPCD with medical therapy including biologics offering limited temporising effect. For those predominantly asymptomatic patients, no intervention can be a suitable management option.

\section{P146 PROVISION OF CARE FOR PREGNANT WOMEN WITH IBD IN THE UK - THE CURRENT LANDSCAPE}

Emma Moore, Sarah Wolloff, Tracey Glanville, Christian Selinger* . Leeds Teaching Hospitals Nhs Trust, Leeds, UK

\subsection{6/gutjnl-2020-bsgcampus.221}

Introduction Inflammatory Bowel Disease (IBD) results in increased rates of negative pregnancy outcomes; particularly in poorly controlled disease. We aimed to describe the current landscape of provision of antenatal care for women with IBD in the UK.

Methods This cross-sectional study collected data on service set-up, on principles of care pre, during, and after pregnancy and on perceived responsibilities of clinicians. An online survey was distributed to all gastroenterology units in the United Kingdom.

Results Data were provided for 97 of 273 IBD units. Prepregnancy counselling was not available in $7 \%$, offered routinely $(39 \%)$, or on request $(54 \%)$ but predominantly $(91 \%)$ in an ad-hoc fashion rather than in a dedicated set-up. $92 \%$ of units provided a nominated gastroenterology consultant during pregnancy. In $86 \%$ of this was the patient's usual consultant rather than a consultant with expertise in pregnancy (14\%).

Combined clinics with obstetricians and gastroenterologists were offered in $14 \%$ of units only, but more often in academic rather than district hospitals $(24 \%$ versus $7 \%$; $\mathrm{p}=0.043$ ) Otherwise, communication with obstetrics was 'as and when required' in $51 \%$ of cases. Patients were reviewed either every trimester (55\%), monthly (15\%) or 'only when required' $(30 \%)$.

The majority of respondents thought gastroenterologists should be involved in decisions regarding routine vaccinations (70\%), breastfeeding (80\%), folic acid dosage (61\%), and VTE prophylaxis $(53 \%) .65 \%$ of participants thought that gastroenterologists should be involved in decision-making regarding delivery method. Regarding IBD indications for elective caesarean section $94 \%$ of participants recommended these for active peri-anal disease, $30 \%$ for previous but healed peri-anal disease, $56 \%$ for ileo-anal pouch and $20 \%$ for previous abdominal and/or pelvic surgery.

Conclusion In a nationwide survey on the provision of antenatal IBD care we found considerable variation in all aspects of service provision, particularly the availability of expertise and specialist services. We have detected areas of poor care (review only when required, communication ad-hoc only, poor provision of dedicated pre-pregnancy counselling). A significant minority of IBD units also felt that they do not need to 